\title{
Erratum
}

\section{Erratum: Costa et al., "Primate Orbitofrontal Cortex Codes Information Relevant for Managing} Explore-Exploit Tradeoffs"

In the article "Primate Orbitofrontal Cortex Codes Information Relevant for Managing Explore-Exploit Tradeoffs" by Vincent Costa and Bruno Averbeck, which appeared on pages 2533-2561 of the March 18, 2020 issue, the authors would like to address the following error.

On page 2556, in Figure 2E, the line colors associated with the labels "Best" and "Worst" were mistakenly switched. The gold line should be orange and labeled "Best" and the orange line should be gold and labeled "Worst." The data plotted are correct. The corrected figure appears below and the article has been updated online (Fig. 2).

DOI: 10.1523/JNEUROSCI.1539-20.2020
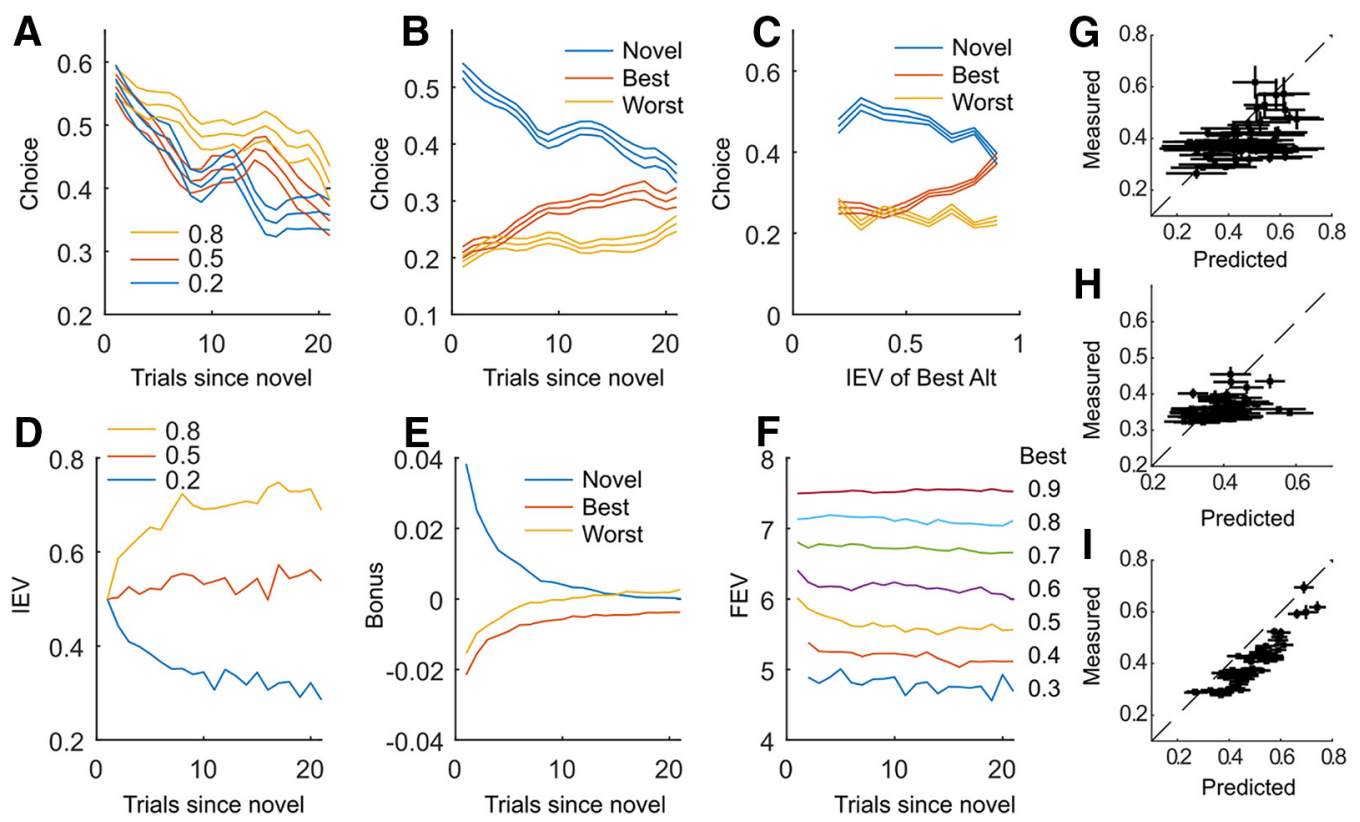

Figure 2. 